\title{
Poly(vinylidene fluoride) composites with carbon nanotubes decorated with metal nanoparticles
}

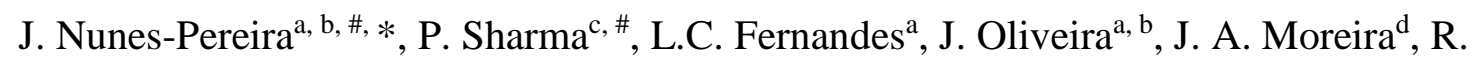
K. Sharma ${ }^{\mathrm{c}}$ and S. Lanceros-Mendez ${ }^{\mathrm{a}, \mathrm{f}, \mathrm{g}, *}$

${ }^{\text {a }}$ Centro de Física, Universidade do Minho, 4710-057 Braga, Portugal

${ }^{\mathrm{b}}$ C-MAST - Centre for Mechanical and Aerospace Sciences and Technologies, Universidade da Beira Interior, 6200-001 Covilhã, Portugal

${ }^{c}$ Department of Chemistry, Indian Institute of Technology Jodhpur, Rajasthan, India

d Algoritmi Research Center, Universidade do Minho, Campus de Azurém, 4800-058

Guimarães, Portugal

e IFIMUP and IN-Institute of Nanotechnology, Department of Physics and Astronomy, Faculty of Sciences, University of Porto, Rua do Campo Alegre, 678, Porto, Portugal

${ }^{\mathrm{f}}$ BCMaterials, Parque Científico y Tecnológico de Bizkaia, 48160-Derio, Spain

g IKERBASQUE, Basque Foundation for Science 48013 Bilbao Spain

*Corresponding Author: jpereira@ fisica.uminho.pt; senentxu.lanceros@bcmaterials.net \#Equal contribution

Keywords: CNT decoration; metal nanoparticles; polymer composites.

\begin{abstract}
:
Polymer composites based on poly(vinylidene fluoride) (PVDF) filled with carbon nanotubes (CNT) decorated with metal nanoparticles (NP) of cobalt, nickel, platinum and palladium have been produced. The CNT nanofillers decorated with metal NP were synthesized by a modified wet impregnation method and their structural, morphological and thermal properties were evaluated. The metal NP ranging from 2 to $10 \mathrm{~nm}$ were found well dispersed on the CNT structure. The structural, optical, thermal and electrical properties of the metal/CNT/PVDF composite films demonstrate that that the inclusion of the nanofillers leads to the nucleation of the $\gamma$-PVDF phase (up to $90 \%$ ) and enhance the thermal properties (higher melting point) of the polymer. The nanofillers also proved to be suitability to tailor the optical properties of the polymer composite films and lead to an increase in the d.c. electrical conductivity (up to $10^{-13}$
\end{abstract}


$\mathrm{S} / \mathrm{cm})$. Thus, the reported metal/CNT nanofillers are suitable to tune PVDF polymer properties towards specific applications.

Keywords: Piezoelectric polymers; PVDF; carbon nanotubes; smart materials; electrical properties

\section{Introduction}

Carbon nanotubes (CNT) are regarded as one of the most suitable reinforcing material for the development of polymer composites due to their excellent electrical, thermal, optical and mechanical properties, as well as their high aspect and surface to-volume ratios $[1,2]$. CNT are chemically very stable regardless the tubes are open and/or filled, opening the possibility to be filled or decorated with metals, semiconductors, salts, organic materials, or therapeutic agents, among others [1]. The combination of CNT and nanoparticles (NP) allow integration and synergetic effects between both materials, giving rise to novel materials with important features for catalysis and nanotechnology [3].

Thus, important efforts are being devoted to the investigation of CNT functionalization in order to develop applications related with solar cells, fuel cells, chemo/biosensors, hydrogen storage, catalysis and drug delivery, among others. The functionalization of the CNT with metal NP gained special attention with the major challenge of decorating uniformly CNT walls [4]. Typically, the two main approaches to the synthesis of metalfilled and decorated CNT are the in situ approach where the filling or decoration procedure is performed during CNT growth, and the ex-situ or post-growth approach, in which the filling is achieved at a later time [1].

Several metal NP and CNT composites have been explored for different purposes. CNT decorated with platinum $(\mathrm{Pt})$ nanoparticles were efficiently synthesized trough an organic colloidal process without aggregation [5]. The composites were suitable to the production of electrochemical sensors with high sensitivity [6]. Carbon materials loaded with faceted Pt nanocrystals [7] exhibited exceptional activity and recyclability for asymmetric hydrogenation of $\alpha$-ketoesters, similarly to Pt nanoparticles/CNT composites efficiently used for asymmetric heterogeneous hydrogenation of methyl pyruvate with highest enantioselectivity [8]. Palladium (Pd) nanoparticles were also used to decorate CNT for hydrogen $(\mathrm{H})$ storage. The composites, produced by a 
solventless method, showed higher hydrogen absorption capacity than pristine CNT which was ascribed to the higher binding energy between Pd and $\mathrm{H}$ atoms [9]. Nickel (Ni) nanoparticles were used to decorate CNT by thermal evaporation, and the formation of $\mathrm{Ni}-\mathrm{C}$ bonds by chemical reaction was found at the interface of the two materials. These CNT showed increased metallic characteristics and proved to be suitable candidates as a template to produce metal nanowires [10]. CNT decorated with nanoparticles of cobalt $(\mathrm{Co})$, iron $(\mathrm{Fe})$ or $\mathrm{Ni}$ were synthesized by a simple chemical method and successfully tested for lightweight microwave adsorption materials in Sband due to the suitable combination of the complex permeability and permittivity from the magnetic nanoparticles [11]. For a similar purpose, Co nanoparticles have also been used to decorate CNT, by hydrothermal treatment, obtaining composites with higher electromagnetic absorption compared to pristine CNT, suggesting an effective way to produce high-efficiency electromagnetic wave absorption materials [12]. Bimetallic nanoparticles based on $\mathrm{Pt}$ with $\mathrm{Ni}, \mathrm{Fe}$ and $\mathrm{Co}$, prepared by chemical reduction, were used to decorate CNT by oxidation for electrochemical storage of hydrogen, showing promising properties as electrocatalyst for high-performance fuel cell applications [13]. For several of the aforementioned applications, it is suitable to integrate those materials into polymer matrices, allowing easier handling and recuperation, as well as simpler integration into devices. Thus, CNT have been introduced into different polymer matrices, including polypropylene [14, 15], styrene-butadiene-styrene [16], epoxy resin [17], and poly(vinylidene fluoride) (PVDF) [18, 19], among others, that have been used to enhance significantly the properties of the polymer and also to broaden the range of application for the polymer itself. Among polymers, PVDF is one of the most representative of a family of polymers with electroactive properties, being therefore one of the polymers of choice for advanced technological applications. PVDF is a semicrystalline polymer with high dielectric constant and electroactive response, including piezoelectric, pyroelectric and ferroelectric properties, which are of large importance for the development of several applications such as sensors, actuators, energy storage and power generation systems, among others [20-23]. PVDF can present five distinct phases with different chain conformations, however the most widely used for applications are the $\alpha, \beta$ and $\gamma$ phases, being the latter two the ones with piezoelectric response [24]. As the combination of decorated CNT and piezoelectric polymers will allow the development of novel multifunctional materials, the present work reports on the 
synthesis and characterization of several metal decorated CNT and the preparation and characterization of the corresponding PVDF matrix composites.

\section{Experimental details}

\subsection{Materials}

The carbon nanotubes (CNT, > $95 \%$ carbon) and chloroplatinic acid (37.50\% Pt basis) were purchased from Aldrich. Nickel nitrate hexahydrate (99\%), cobalt nitrate hexahydrate (> $99 \%$ ) and palladium nitrate hexahydrate (> $95 \%$ ) were obtained from Acros organics. Poly(vinylidene fluoride) (PVDF), Solef ${ }^{\circledR} 1015$ was acquired from Solvay, and the solvent N,N-dimethylformamide (DMF) was supplied by Flucka.

\subsection{Fillers synthesis}

A modified wet impregnation method was carried out for metal loaded CNT decoration [8] (Fig. 1). In this procedure, the CNT were refluxed under constant stirring in $\mathrm{HNO}_{3}$ (68 wt $\%$ ) at $140{ }^{\circ} \mathrm{C}$, and extracted by centrifugation at $3000 \mathrm{rpm}$. CNT were washed several times with water followed by ethanol. Resultant activated CNT were dried at 80 ${ }^{\circ} \mathrm{C}$ for $4 \mathrm{~h}$ under argon and then immersed in an ethanolic solution under stirring and sonication, simultaneously. Then, the corresponding metal salt was dissolved in degassed ethanol and added to the CNT solution slowly via syringe pump under hydrogen bubbling for a period of $12 \mathrm{~h}$. The solution was stirred for another $24 \mathrm{~h}$ at room temperature and finally heated at $100{ }^{\circ} \mathrm{C}$ for $12 \mathrm{~h}$. Metal nanoparticles were tinted on carbon surface, the metal decorated CNT were isolated via centrifugation, followed by washing with deionized degassed water and dried at $80{ }^{\circ} \mathrm{C}$ for $16 \mathrm{~h}$ under argon.

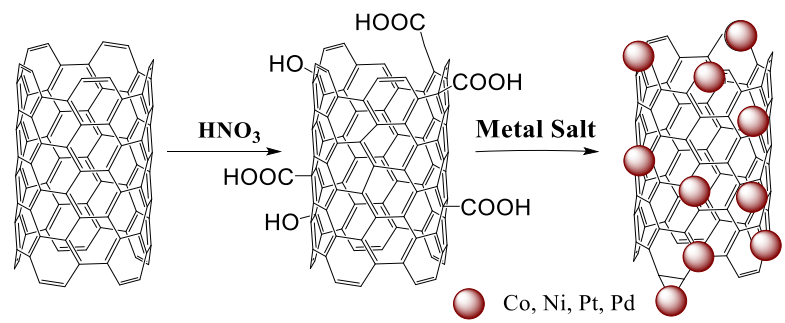

Fig. 1. Schematic representation of a typical decoration process of CNT.

Following this procedure, the following decorated CNT were prepared: Pd/CNT, Ni/CNT, Co/CNT, Pt(in), Pt(out) and Pt-Co. 


\subsection{Fillers characterization}

$\mathrm{X}$-ray diffraction (XRD) performed in an X-ray diffractometer, D8 advance (Bruker, U.S.A.) using $\mathrm{Cu} \mathrm{K \alpha 1}(\lambda=1.54056 \AA)$ as a radiation, source was used to ascertain the quality and crystalline nature of carbon materials with tube current and voltage $40 \mathrm{~mA}$ and $40 \mathrm{kV}$, respectively.

The morphology of metal/CNT nanofillers was determined using scanning electron microscopy (SEM) EVO18 Ziess with an accelerating voltage of $20 \mathrm{KeV}$. Metal nanoparticles were confirmed by energy dispersive X-ray (EDX) spectroscopy. Transmission electron microscopy (TEM) was carried out in a FEI Tecnai-G2 T20. For TEM study, the materials were dispersed in ethanol using tip ultrasonication. The samples were prepared by placing a droplet of suspension onto a copper coated grid and dried in air. The TEM analysis articulate shape and distribution of the nanoparticles on carbon support.

Thermogravimetric analyses (TGA) were performed with a TGA-6000 thermal analyzer (Perkin Elmer) under a nitrogen atmosphere (flow $19.8 \mathrm{~mL} / \mathrm{min}$ and pressure $3 \mathrm{bar}$ ) and a heating rate of $10^{\circ} \mathrm{C} / \mathrm{min}$.

\subsection{Films preparation}

First, the decorated CNT were dispersed in a solution of DMF in an ultrasonic bath during $6 \mathrm{~h}$. PVDF was then added to the solution to obtain a concentration of $\mathrm{PVDF} / \mathrm{DMF}$ of $10 / 90 \mathrm{wt} \%$ and the fillers to polymer relative concentration ranged from 0.1 to $0.5 \mathrm{wt} \%$. The solution was then magnetically stirred at $40{ }^{\circ} \mathrm{C}$, until complete polymer dissolution $(\sim 2 \mathrm{~h})$. Then, the mixture was placed in clean glass substrate and spread with a doctor blade to achieve a thickness of $450 \mu \mathrm{m}$. Finally, the films were placed in an oven at $210^{\circ} \mathrm{C}$ during 10 min to complete solvent evaporation and polymer melting in order to erase composite preparation history and to obtain non-porous films [25]. In the following, the polymer composite samples will be identified by the metal nanoparticle decorating the CNT (Pd, Ni, Pd, Pt(in), Pt(out) and Pt-Co) and the respective amount when necessary.

\subsection{Films characterization}

The polymer phase in the composites was evaluated by Fourier transformed infrared spectroscopy (FTIR) on a Jasco FT/IR-4100 in attenuated total reflectance (ATR) mode; 32 scans were performed at room temperature between 4000 to $600 \mathrm{~cm}^{-1}$ with a 
resolution of $4 \mathrm{~cm}^{-1}$. The relative fraction of the $\gamma$-phase $(\mathrm{F}(\gamma))$ in the samples was calculated according to:

$\mathrm{F}(\gamma)=\frac{\mathrm{A}_{\gamma}}{\left(\mathrm{K}_{\gamma} / \mathrm{K}_{\alpha}\right) \mathrm{A}_{\alpha}+\mathrm{A}_{\gamma}}$

where $A_{\alpha}$ and $A_{\gamma}$ are the absorbance at 766 and $833 \mathrm{~cm}^{-1}$ respectively, and $\mathrm{K}_{\alpha}(0.365$ $\left.\mu \mathrm{m}^{-1}\right)$ and $\mathrm{K}_{\gamma}\left(0.150 \mu \mathrm{m}^{-1}\right)$ are the absorption coefficients at the respective wavenumber [24].

The optical properties of the composites were measured by UV-Vis spectroscopy with a Jasco V-670 spectrophotometer coupled with a sample holder for solids. The spectra were recorded at room temperature from 200 to $900 \mathrm{~nm}$.

Raman spectra were recorded, at room temperature, by using an Olympus microscope and a $50 \times$ objective. For excitation, it was used the $514.5 \mathrm{~nm}$ polarized line of an $\mathrm{Ar}+$ laser, with an incident power of about $16 \mathrm{~mW}$ impinging on the sample. The acquisition time for each measurement was set at $120 \mathrm{~s}$ and two/three scans. The scattered light was analyzed using a T64000 Jobin-Yvon spectrometer, operating in a triple subtractive mode, and equipped with a liquid nitrogen cooled CCD detector, in a Stokes frequency range from 200 to $2000 \mathrm{~cm}^{-1}$ [26]. In order to obtain information regarding the homogeneity of our samples, Raman spectra was recorded at different positions on the sample surfaces.

The thermal properties were evaluated by differential scanning calorimetry (DSC) analysis carried out with a Perkin-Elmer DSC 8000 instrument between 25 and $200{ }^{\circ} \mathrm{C}$ at a heating rate of $20^{\circ} \mathrm{C} \mathrm{min}{ }^{-1}$ under a flowing nitrogen atmosphere. The samples were first placed in aluminum pans of $30 \mu \mathrm{L}$ with perforated lids to allow the release of volatiles. The degree of crystallinity $\left(\chi_{c}\right)$ of each sample was calculated by equation 2 :

$\chi_{\mathrm{c}}=\frac{\Delta \mathrm{H}_{\mathrm{f}}}{\Delta \mathrm{H}_{100}}$

where $\Delta \mathrm{H}_{\mathrm{f}}$ is the melting enthalpy of the sample and $\Delta \mathrm{H}_{100}$ is the melting enthalpy of a $100 \%$ crystalline sample of pure PVDF (104.6 J/g) [27].

The d.c. volume electrical conductivity of the samples was obtained by measuring the characteristic IV curves at room temperature with a Keithley 6487 picoammeter/voltage source. Previously, circular gold electrodes ( $5 \mathrm{~mm}$ diameter) were deposited by sputtering using a Polaron, model SC502 sputter coater (90 s at 20 mbar and 20 mA) on both sides of the films. From the IV characteristics of the samples, the electrical 
conductivity $(\sigma)$ was calculated taken into account the geometrical factors in according to:

$$
\sigma=\mathrm{R} \frac{\mathrm{L}}{\mathrm{A}}
$$

where, $\mathrm{R}$ is the electrical resistance, $\mathrm{L}$ is the sample thickness and $\mathrm{A}$ is the area of the electrodes.

\section{Results and discussion}

\subsection{Nanofillers characterization}

Fig. 2 shows the XRD patterns of the different metal/CNT composites.

a)

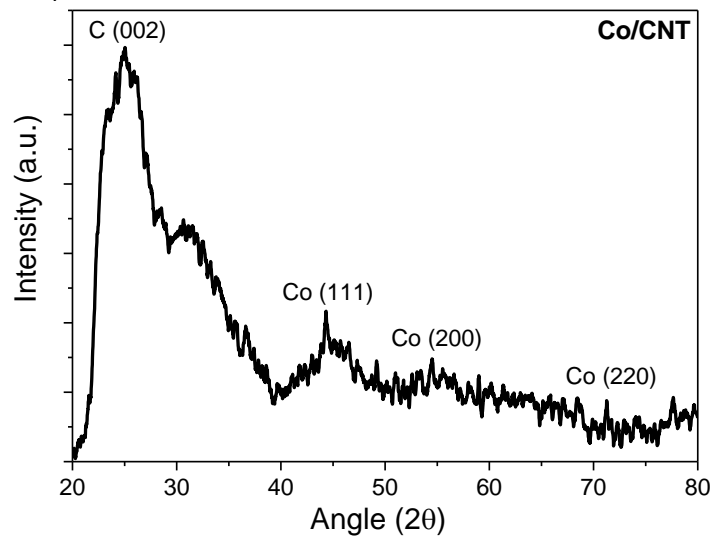

c)

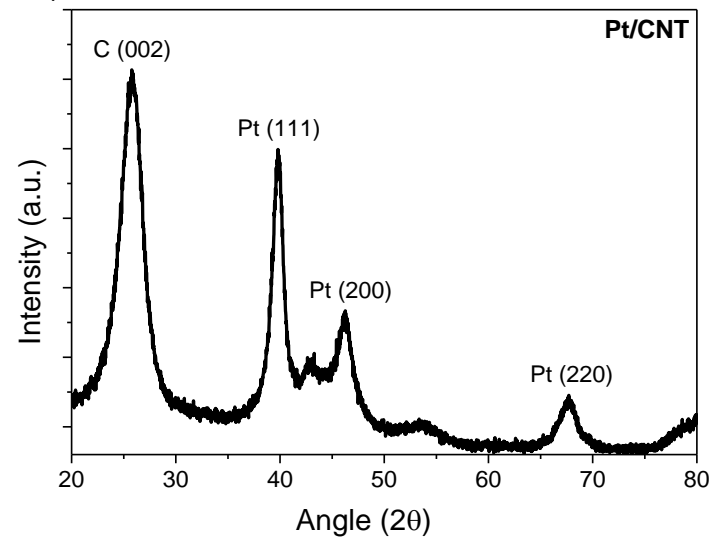

b)

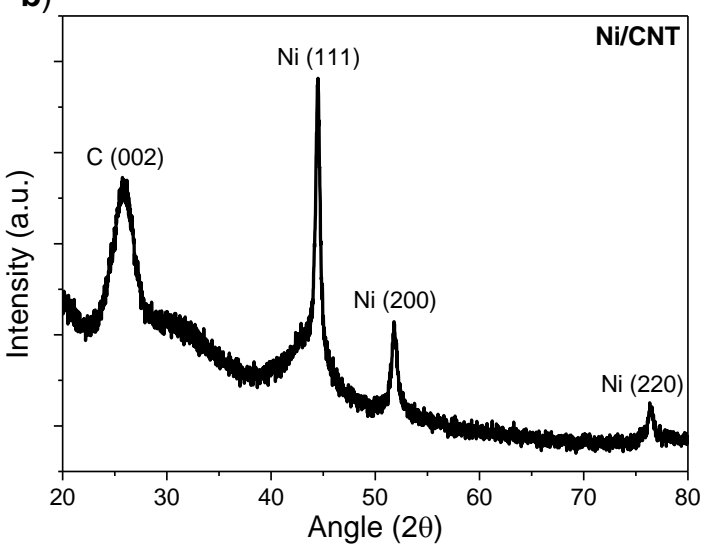

d)

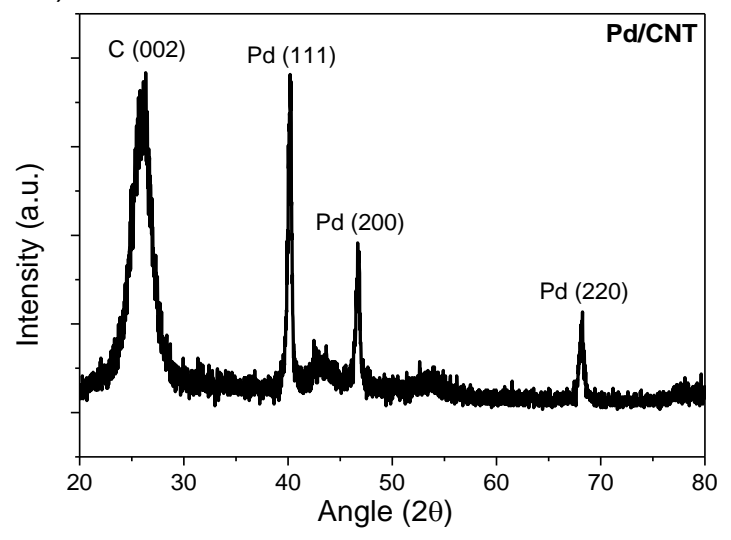

Fig. 2. XRD patterns of a) $\mathrm{Co} / \mathrm{CNT}$; b) Ni/CNT; c) Pt/CNT and d) different loading of Pd/CNT.

The Fig. 2 confirms the metal structure, and thus the decoration, on the CNT. The XRD patterns show face-centered cubic (FCC) crystal structure of metal along with CNT, identified by the peaks at $2 \theta=25.90,42.70$ and 53.50 are ascribed to the (002), (100) and (004) planes respectively. 
For $\mathrm{Co} / \mathrm{CNT}$, the XRD pattern presents peaks at $44.3,54.5$ and 77.6 corresponding to diffractions of the (111), (200) and (220) planes of the FCC lattice of Co nanoparticles (Fig. 2a). Similarly, Ni/CNT shows peaks at 44.5, 51.8 and 76.3 ascribed to the diffraction of FCC planes of Ni (Fig. 2b). In the case of Pt/CNT and Pd/CNT (Fig. 2c and d), three major peaks at 39.6, 46.4 and 67.7 ascribed to the diffractions of the (111), (200) and (220) planes of the FCC lattice of the metal nanoparticles (Pt and Pd). No differences were found between the spectra of $\mathrm{Pt}(\mathrm{in}) / \mathrm{CNT}$ and $\mathrm{Pt}(\mathrm{out}) / \mathrm{CNT}$ nanoparticles, so the spectrum of $\mathrm{Pt} / \mathrm{CNT}$ composite is representative of both.

Fig. 3 presents the SEM images and EDX patterns of the metal/CNT composites.

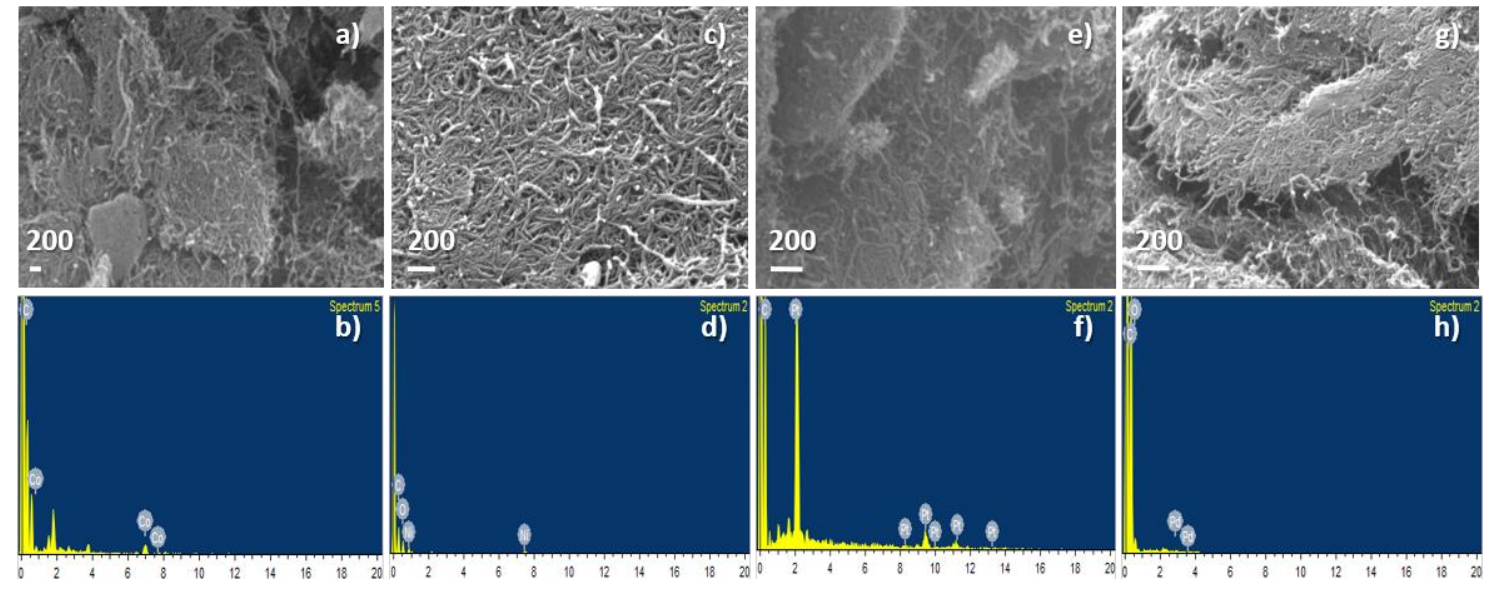

Fig. 3. SEM and EDX analysis of: a) and b) Co/CNT; c) and d) Ni/CNT; e) and f) Pt/CNT; g) and h) $\mathrm{Pd} / \mathrm{CNT}$.

Fig. 3 shows the metal nanoparticles (brighter areas) well dispersed on the CNT structure and the presence of these metal nanoparticles (Co, Ni, Pt and Pd) was confirmed by EDX spectroscopy analysis.

Finally, Fig. 4 shows representative TEM images of the metal/CNT composites on carbon support.
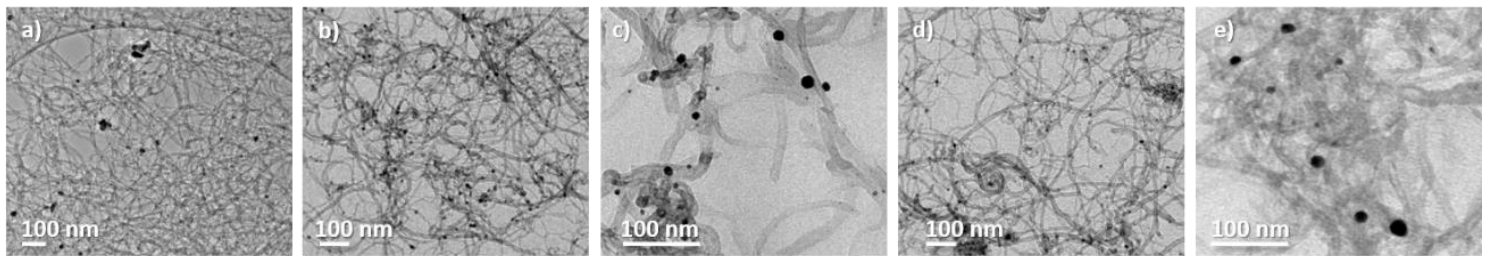

Fig. 4. TEM images of: a) Co/CNT; b) Ni/CNT; c) Pt(in)/CNT; d) Pd/CNT and e) Pt(out)/CNT.

The TEM analysis articulates shape and distribution of the metal nanoparticles on carbon support. The nanoparticle size ranged from 2 to $10 \mathrm{~nm}$ with good dispersion. No free nanoparticles were observed on the background of the TEM images which is a 
strong indication that all the nanoparticles are attached to the CNT. Regarding CNT, the TEM images allow observing their tubular shape with an outside diameter around $11 \pm$ $2 \mathrm{~nm}$ and inner diameter of $4 \pm 1 \mathrm{~nm}$. It is noteworthy to notice that for Pt(in)/CNT case the metal nanoparticles were found both on the outer and inner surface of the CNT (Fig. $4 c)$ unlike the other cases where metal nanoparticles decorate the outer surface of the CNT (Fig. 4a, b, c and e).

Fig. 5 presents the TGA thermograms of the metal/CNT composites and functionalized CNT.

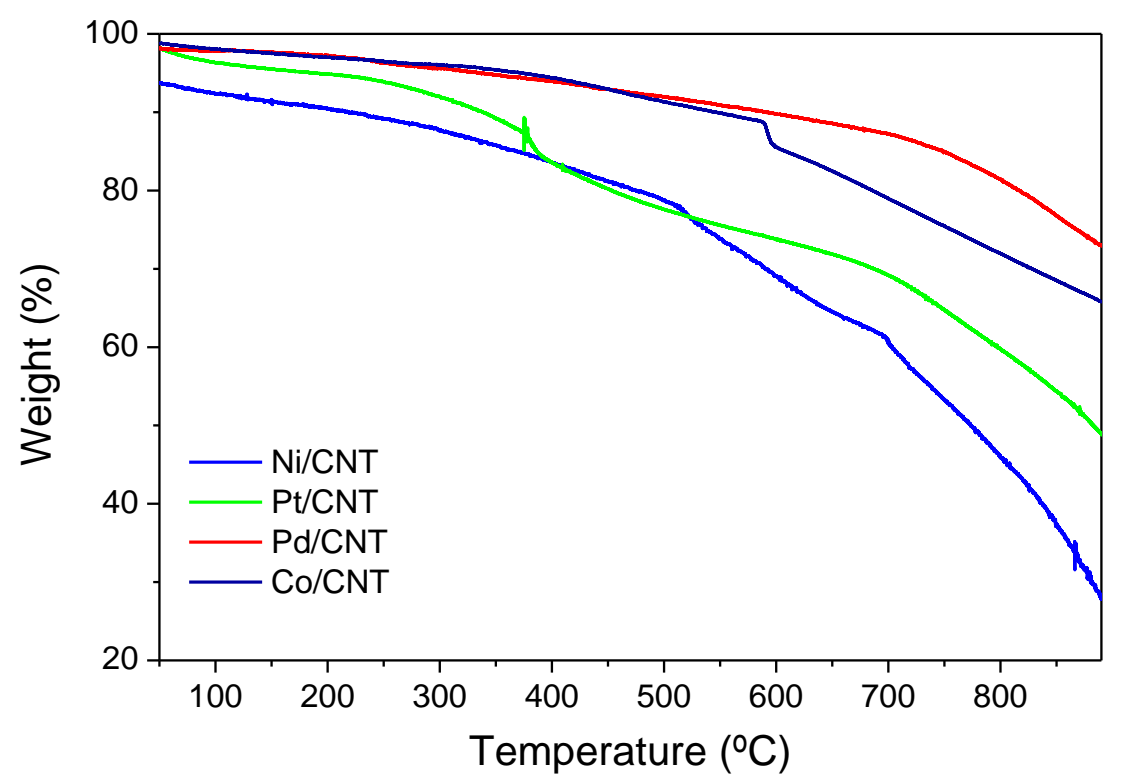

Fig. 5. TGA analysis of the CNT composites decorated with $\mathrm{Co}, \mathrm{Ni}, \mathrm{Pt}$ and $\mathrm{Pd}$.

TGA analysis showed weight decomposition with temperature of the metal decorated CNT composites. The oxidized behavior of the compounds was studied up to $900{ }^{\circ} \mathrm{C}$ under $\mathrm{N}_{2}$. Weight loss below $400{ }^{\circ} \mathrm{C}$ is due to the desorption of moisture and volatile impurities and, at higher temperatures, the carbon material loses most of its starting weight (around $610^{\circ} \mathrm{C}$ ) which increases gradually with increasing temperature of the post-carbonization. The metal/CNT catalysts show a first decomposing step due to moisture, a second one due to inorganic impurities such as metals ( $\mathrm{Co}, \mathrm{Ni}, \mathrm{Pt}$ and $\mathrm{Pd}$ ) and a third one related to carbon oxidation [28]. The thermogram of Pt/CNT is representative for both $\mathrm{Pt}(\mathrm{in}) / \mathrm{CNT}$ and $\mathrm{Pt}$ (out)/CNT composites, since no differences were found between the thermograms. 


\subsection{Films characterization}

\subsubsection{Structural properties}

The effect of the nanofillers in the crystallization kinetics of the polymer was studied by FTIR-ATR and the spectra are shown in Fig. 6 for the different composites films. The quantification of the phase content was performed after equation 1, assuming that the crystalline phase of the polymer is either in the $\alpha$ or $\gamma$-phases with no or small traces of $\beta$-phase, as indicated by the FTIR-ATR spectra.
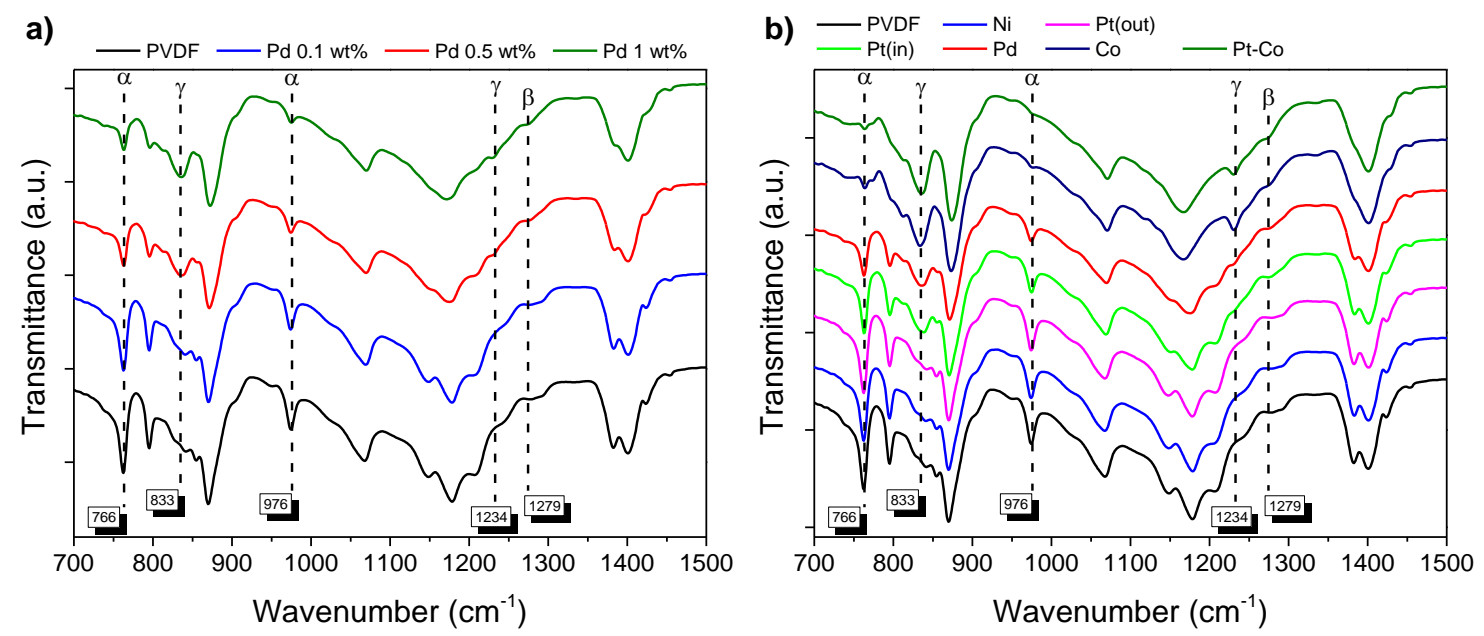

Fig. 6. FTIR-ATR spectra of: a) Pd/CNT/PVDF composites with different nanofillers content and b) all metal/CNT/PVDF composites for $0.5 \mathrm{wt} \%$ nanofiller content.

The spectra of Fig. 6a shows the influence of the Pd/CNT filler content in the structure of the polymer composite. The pristine PVDF film presents the characteristic absorption bands exclusive of the PVDF $\alpha$-phase at 766, 795 and $976 \mathrm{~cm}^{-1}$, as expected by the processing [24]. The inclusion of $\mathrm{Pd} / \mathrm{CNT}$ leads to the reduction in the intensity of the $\alpha$-phase bands with the increasing of filler content and to the appearance and increase of the bands at 833 and $1234 \mathrm{~cm}^{-1}$ characteristic of the $\gamma$-phase; no traces of $\beta$-PVDF were found $\left(1279 \mathrm{~cm}^{-1}\right)$. This indicates that the addition of $\mathrm{Pd} / \mathrm{CNT}$ fillers induces the crystallization of the electroactive $\gamma$-PVDF even at the processing temperature of 210 ${ }^{\circ} \mathrm{C}$, which is a consequence of the electrostatic interactions introduced by the $\mathrm{Pd} / \mathrm{CNT}$ nanofillers into the polymer matrix leading to the rotation of the polymer chains to an all-trans conformation and crystallization of the polymer in a polar phase, similarly to that reported for PVDF composites with Y zeolites [29] and silver nanoparticles [29]. The phase quantification reveals an $\gamma$-PVDF content ranging from $\sim 27$ to $68 \%$ for the samples with 0.1 and $1 \mathrm{wt} \%$ of $\mathrm{Pd} / \mathrm{CNT}$ respectively. 
Fig. $6 \mathrm{~b}$ shows the influence of the different metal/CNT fillers in the structure of the polymer matrix with a content of $0.5 \mathrm{wt} \%$. The spectra of the composites with CNT decorated with Ni, Pt(in) and Pt(out) mainly present the characteristic bands of $\alpha$ PVDF, whereas the samples of the composites with Pd, Co and Pt-Co presents the bands corresponding to $\gamma$-PVDF and $\alpha-P V D F$, the later undergoing a large reduction mainly for Co and Pt-Co composites. Thus, $\gamma$-PVDF contents of $\sim 90$ and $86 \%$ were calculated for the samples with CNT decorated with Pt-Co and Co respectively. It is to notice that the values are among the largest found in the literature, such as 95 and $94 \%$ for PVDF composites with Y zeolite [29] and silver nanoparticles [29] respectively. Fig. 7 shows the Raman spectra of the different metal/CNT/PVDF composites for $0.5 \mathrm{wt} \%$ nanofiller loading.

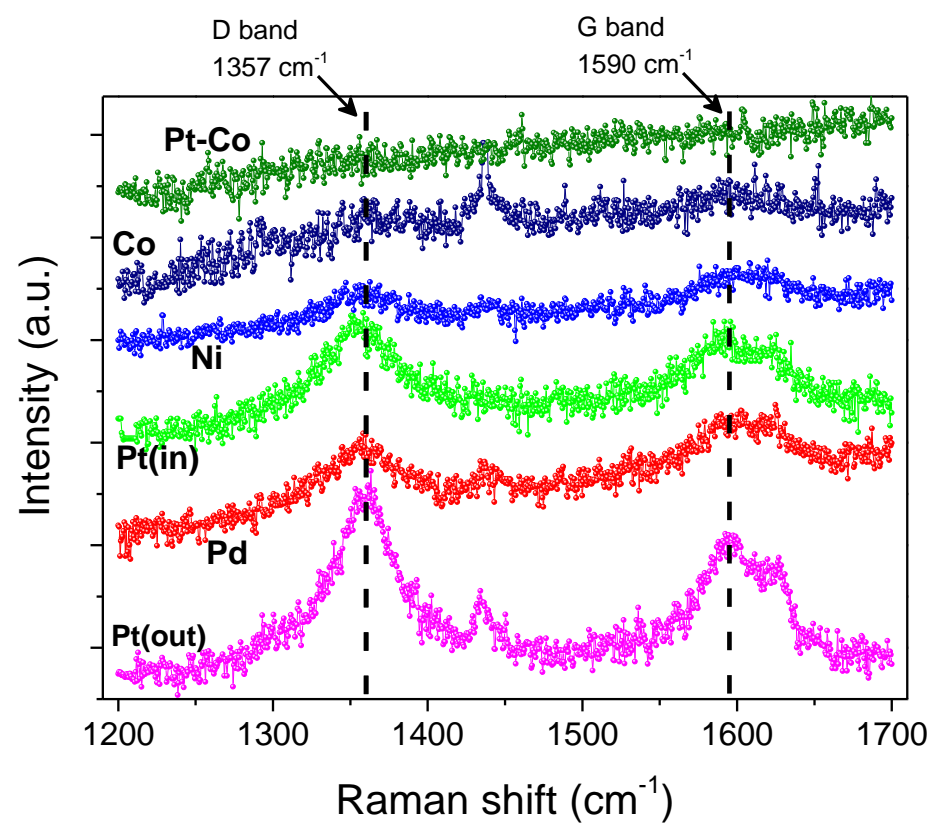

Fig. 7. Raman spectra of several metal/CNT/PVDF composites for $0.5 \mathrm{wt} \%$ nanofillers.

It is possible to identify different behavior groups based on the relationship of the Raman signal and the electrical conductivity. The first group is composed by the samples with higher conductivity, the samples with Pt-Co and Co. It is observed that the two characteristics bands of carbon nanotubes are not well-defined and visible. This fact is due to the high electrical conductivity of the samples that decreases the Raman features. The second group corresponds to the samples with $\mathrm{Ni}, \mathrm{Pd}$ and $\mathrm{Pt}(\mathrm{in})$ that present Raman spectra with perceptible but not clearly defined bands namely for $\mathrm{Ni}$ and $\mathrm{Pd}$, being in agreement with the intermediate state of conductivity. The third group corresponds to the sample decorated with $\mathrm{Pt}$ (out) nanoparticles, in which is possible to 
distingue two different and well defined bands. The sample with the highest bands in the Raman spectra corresponds to the sample with the lowest conductivity. These bands indicated as D and G, observed around 1357 and $1587 \mathrm{~cm}^{-1}$, respectively, are characteristic of the carbon nanotube structure which corresponds to the sp3 and sp2 hybridized state of carbon, respectively [30]. The decrease of the D band is due to the disorder induced by the doped metal nanoparticles and is attributed to the presence of disorder and defects in the crystalline structure of carbon systems [31]. On the other hand, the decrease of the $\mathrm{G}$ band is due to the interaction of the metal nanoparticles with the CNT, which leads to the conversion of $\mathrm{sp}$ to $\mathrm{sp} 2$ carbon $[30,32]$. The intensity ratio of $\mathrm{D}$ peak to the $\mathrm{G}$ peak, identified as the ID/IG ratio, is proportional to the amount of system disorder in the sample [32]. It is also worth highlighting that is possible to found, for the lower conductive samples, some bands near the $G$ band ( $\mathrm{D}^{\prime}$ band). This provides indication of the number of structural defects and disorder present in the material. Pt(out) composites show the highest ID/IG ratio, which is higher than the $\mathrm{Pt}($ in) and significantly higher than the one observed for $\mathrm{Ni}$ and for Pd [33]. The band at around $1437 \mathrm{~cm}^{-1}$ is well defined and visible for some samples such as $\mathrm{Pd}$ and Co, which could be related to the disorder and the presence of defects caused by the C-H bending [34].

\subsubsection{Thermal and optical properties}

Fig. 8 shows the DSC thermograms of the samples and table 1 summarizes the degree of crystallinity calculated through equation 2 . 
a)

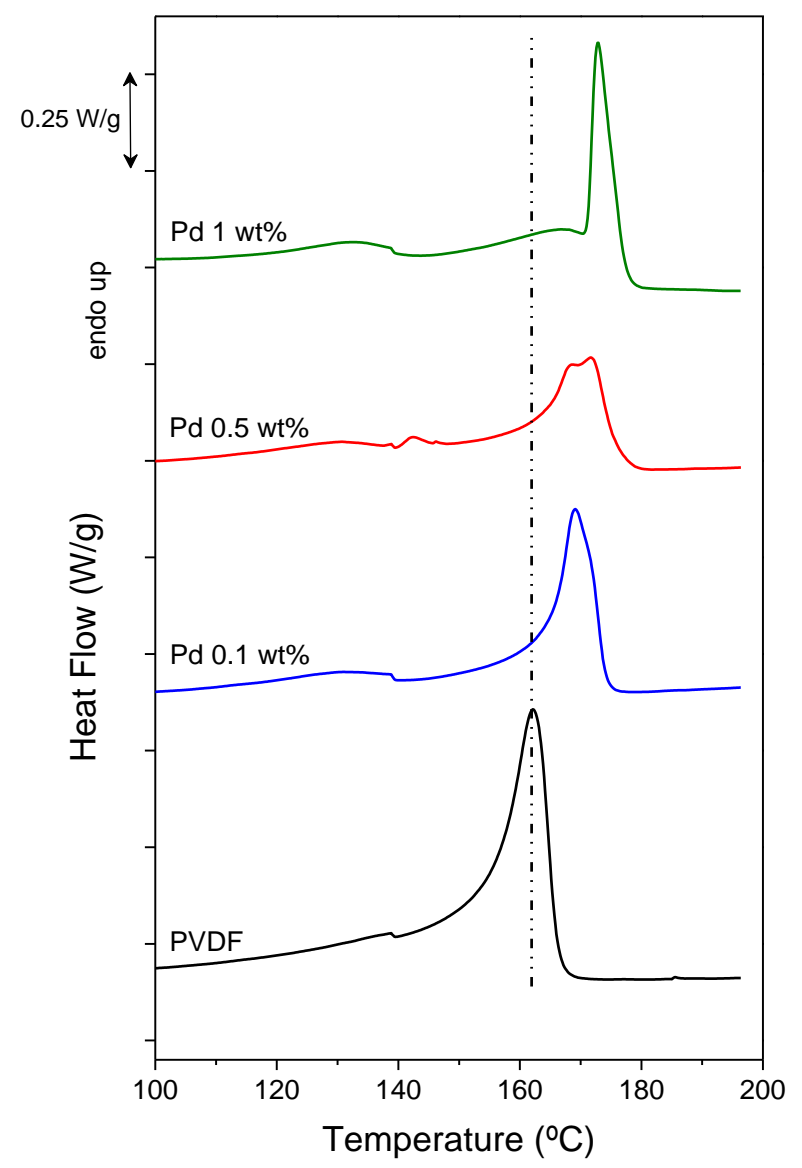

b)

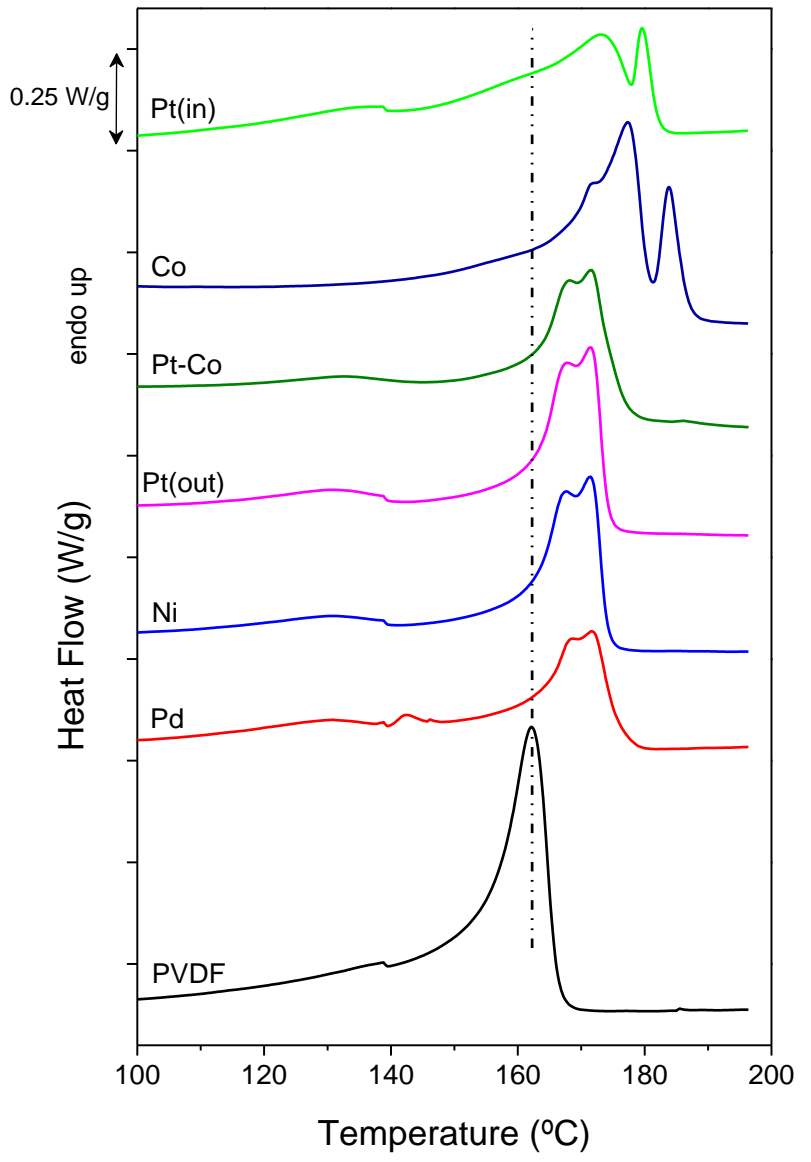

Fig. 8. DSC thermograms of: a) Pd/CNT/PVDF composites with different nanofiller contents and b) all metal/CNT/PVDF composites for $0.5 \mathrm{wt} \%$ nanofiller content.

Table 1

Degree of crystallinity $\left(\chi_{c}\right)$ of the all polymer composites.

\begin{tabular}{ll}
\hline Sample & $\chi_{\mathrm{c}}(\boldsymbol{\%}) \mathbf{\mathbf { 3 } \%}$ \\
\hline PVDF & 36 \\
Pd $0.1 \mathrm{wt} \%$ & 19 \\
Pd $0.5 \mathrm{wt} \%$ & 21 \\
Pd $1 \mathrm{wt} \%$ & 25 \\
Ni & 32 \\
Co & 36 \\
Pt(in) & 35 \\
Pt(out) & 33 \\
Pt-Co & 30 \\
\hline
\end{tabular}

Fig. 8a shows the thermogram of the pristine film compared to the composites with 0.1 , 0.5 and $1 \mathrm{wt} \%$ of Pd. The thermogram of the PVDF film presents a high endothermic peak with a maximum heat flow around $162{ }^{\circ} \mathrm{C}$ ascribed to the melt of the crystalline $\alpha$ phase of the polymer [24]. In turn, compared to the pristine sample, the thermograms of 
the composites show a shift in the melting peak of about 8 to $10{ }^{\circ} \mathrm{C}$ towards higher temperatures which is indicative of the presence of $\gamma-\mathrm{PVDF}$, and is in agreement with the literature on the $\gamma$ phase of PVDF [24]. This shift tends to increase with increasing filler content, higher for the sample with $1 \mathrm{wt} \%$ of $\mathrm{Pd}$, with a maximum heat flow around $173^{\circ} \mathrm{C}$.

Further, Fig. 8b presents the thermograms of the pristine PVDF sample and all composites with a filler content of $0.5 \mathrm{wt} \%$. All composites show a shift in the thermogram towards higher temperatures, compared to pure polymer. It is to notice that the composites with $\mathrm{CNT}$ decorated with $\mathrm{Pd}, \mathrm{Ni}, \mathrm{P}(\mathrm{out})$ and $\mathrm{Pt}-\mathrm{Co}$ present a shoulder in the melting peak and the composites with CNT decorated with Co and Pt(in) present two separated peaks confirming in both cases the presence of a phase with higher thermal stability than $\alpha$ phase: the $\gamma$ phase [35].

Table 1 shows that the inclusion of the fillers generally results in a slight decrease of the degree of crystallinity for all samples from $36 \%$ for pristine PVDF up to a minimum of $19 \%$ for the sample with $0.1 \mathrm{wt} \%$ of $\mathrm{Pd}$, although the differences are within the experimental error for some samples, this trend indicates that the presence fillers induces a defective crystallization of the polymer at the interfaces with the fillers, leading also to changes in nucleation and growth kinetics [36]. The samples with Pd nanofillers present a degree of crystallinity slightly lower compared to the remaining composites, and the increasing of the filler content from 0.1 to $1 \mathrm{wt} \%$ results in an increase of the crystallinity from to 19 up to $25 \%$, suggesting that for concentrations higher than $0.1 \mathrm{wt} \%$ this filler acts as a nucleating agent for the crystallization of the polymer matrix [37].

The Fig. 9 shows the UV-Vis spectra of the samples. 

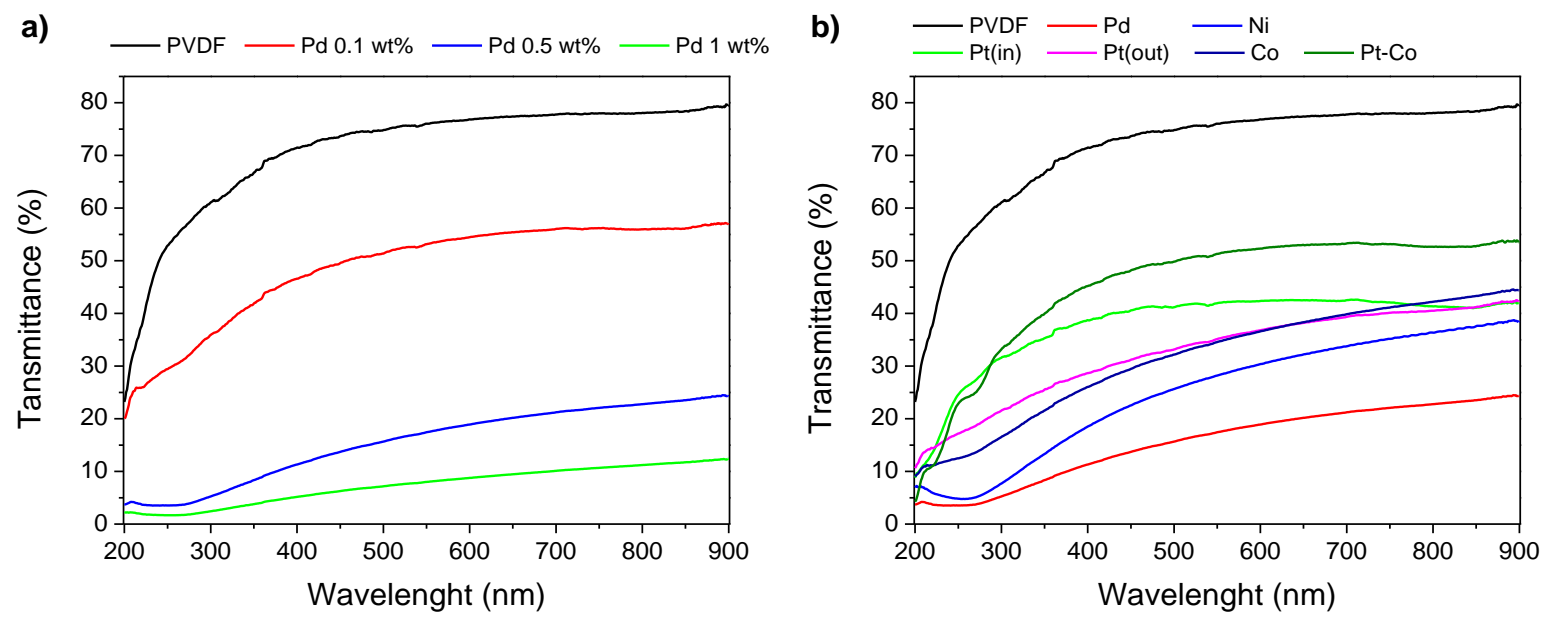

Fig. 9. UV-Vis spectra of: a) Pd/CNT/PVDF composites with different filler content; b) the different metal/CNT/PVDF composites for $0.5 \mathrm{wt} \%$ nanofiller content.

The spectra of Fig. 9a shows that the pristine film of PVDF shows a transmittance between 70 and $80 \%$ in the range between 400 to $700 \mathrm{~nm}$. The inclusion of $\mathrm{Pd} / \mathrm{CNT}$ decreases the transmittance proportionally to filler content up to a minimum value of 5$10 \%$ in the visible spectral range for the sample with the highest $\mathrm{Pd} / \mathrm{CNT}$ content of 1 wt\%. Fig. 9b shows that all composite films present lower transmittance than the pristine polymer film. The sample of Pt-Co shows the higher transmittance value among the composites (40 to $50 \%$ in the visible region of the spectrum), in turn the Pd film shows the lowest transmittance (between 10 and $20 \%$ ). Further, all the composites exhibit considerable UV shielding, over the UVA, UVB and UVC regions (200-400 $\mathrm{nm})$. The UV-Vis result show that the metal/CNT fillers allow to tailor the optical properties of the PVDF composite film varying filler concentration and/or type, in order to match specific application requirements, thus taking advantage of the unique optical properties of the fillers [38].

\subsubsection{Electrical properties}

The effect of the metal/CNT filler type and content on the d.c. electrical conductivity was evaluated by the analysis characteristic IV curves of the films. Fig. 10 presents the characteristic IV curves of the composites and the filler type and content dependence of the d.c. electrical conductivity. 

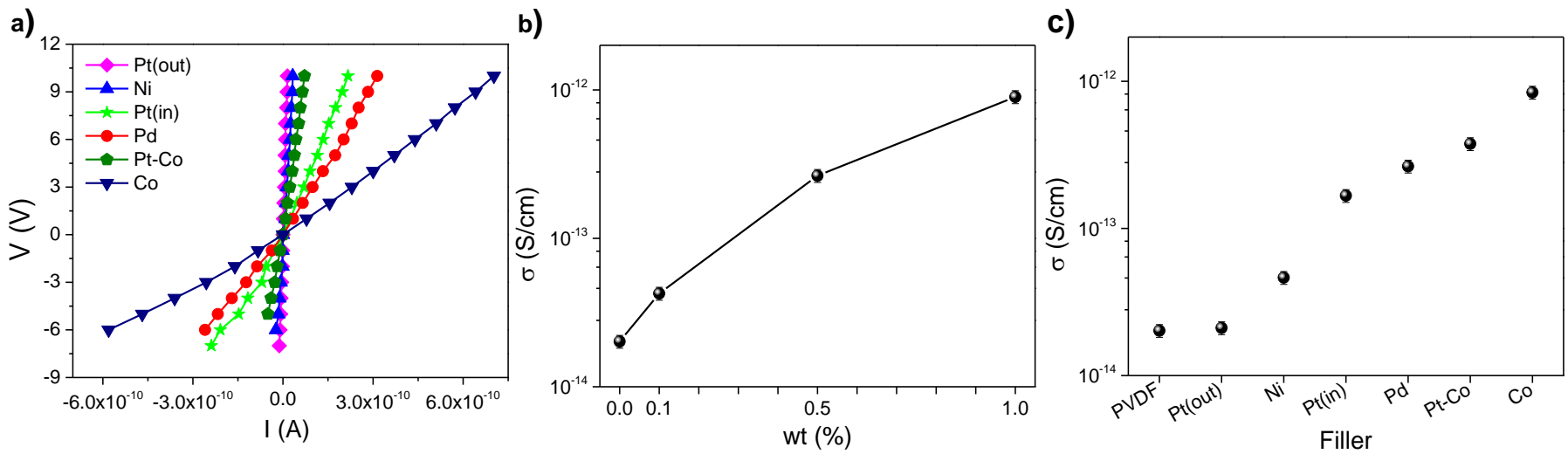

Fig. 10. a) IV curves for the different composites with $0.5 \mathrm{wt} \%$ metal/CNT content; d.c. electrical conductivity of b) Pd/CNT/PVDF composites for different filler concentrations and c) the different metal/CNT/PVDF composites for $0.5 \mathrm{wt} \%$ nanofiller content.

Fig. 10a show the characteristic IV curves of the composites with $0.5 \mathrm{wt} \%$ content of decorated CNT. All samples show a good linearity between current and voltage (ohmic behavior) allowing to calculate the d.c. electrical conductivity through the application of the Ohm's law (equation 3). Fig. 10b shows that the d.c. electrical conductivity of the composite depends on the concentration of the $\mathrm{Pd} / \mathrm{CNT}$, increasing with the increasing nanofiller content by an order of magnitude, ranging from $10^{-14} \mathrm{~S} / \mathrm{cm}$, for pristine PVDF, up to $10^{-13} \mathrm{~S} / \mathrm{cm}$ for Pd/CNT/PVDF sample with $1 \mathrm{wt} \%$ filler content. With respect to the influence of filler type in the d.c. electrical conductivity it is observed (Fig. 10c) that the CNT decorated with Pt(out) do not affect the conductivity of the samples, which is related with the strong disorder observed in the Raman spectra (Fig. 6) for this nanofillers, that shows the highest ID/IG ratio, and reduces the electrical conductivity of the CNT. The inclusion of Ni nanoparticles decorated CNT does not affect significantly the d.c. electrical conductivity of the composite registering just a slight increase within the same order of magnitude $\left(10^{-14} \mathrm{~S} / \mathrm{cm}\right)$, while the samples with $\mathrm{Pt}$ (in), Pd and Pt-Co lead to an increase of one order of magnitude. The sample decorated with Co nanoparticles shows the maximum increase in the electrical conductivity, of almost two orders of magnitude, achieving the value of $\sim 8.4 \times 10^{-13}$ $\mathrm{S} / \mathrm{cm}$. These results are in accordance with the Raman results for the samples with Co that presents spectra without visible D band, indicating weak disorder in the CNT system. Despite all composites present higher conductivity than the pristine PVDF sample, the increase of one order of magnitude from $10^{-14}$ to $10^{-13} \mathrm{~S} / \mathrm{cm}$ originated by the addition of the metal/CNT is significantly lower than that reported for composites of PVDF with CNT at similar nanofillers concentrations $[39,40]$. This suggests that the 
decoration of the CNT with metal nanoparticles induce defective structures influencing the $\pi$ orbitals of the CNT leading to the decrease of the conductivity [41].

\section{Conclusions}

CNT were successfully decorated with several metal nanoparticles (Co, Ni, Pt and Pd) by a modified wet impregnation method. The presence of the metal nanoparticles in the CNT structure was verified and a good dispersion of nanoparticles ranging from 2 to 10 nm was observed. PVDF composites with the different metal decorated CNT were prepared by melt casting method. The metal decorated CNT promote the nucleation of the $\gamma$-PVDF electroactive phase up to $\sim 90 \%$ and the CNT decorated with Pt(out) leads to the highest system disorder. The metal/CNT nanofillers also enhance the thermal properties by increasing the melting temperature around $10{ }^{\circ} \mathrm{C}$ and result in a decrease of the degree of crystallinity of the samples, from $\sim 36 \%$ to $19 \%$ for pristine PVDF and $\mathrm{Pd} / \mathrm{CNT} 0.1 \mathrm{wt} \%$ respectively. Further, metal/CNT nanofillers allow tailoring the optical properties of PVDF films from transmittances of 70 to $80 \%$ (PVDF) down to 5 to $10 \%$ (composites) depending on nanofiller type or content. Finally, the inclusion of the nanofillers results in a decrease of the d.c. electrical conductivity by one order of magnitude from $10^{-14} \mathrm{~S} / \mathrm{cm}$ (PVDF) to $10^{-13} \mathrm{~S} / \mathrm{cm}$ (composites). In summary, these results show that filler type or concentration allows to tune the PVDF composite film properties for specific applications, such as nucleating the piezoelectric $\gamma$-phase, for sensor and actuator application, and tuning crystallinity and optical transmittance. Further, the catalytic activity of the metal decorated CNT allows to further expand the range of applicability of the PVDF composites to new areas.

\section{Acknowledgements}

This work was supported by the Portuguese Foundation for Science and Technology (FCT) in the framework of the strategic project UID/FIS/04650/2013, by FEDER funds through the COMPETE 2020 - Programa Operacional Competitividade e Internacionalização (POCI) with the reference project POCI-01-0145-FEDER-006941. JNP wish to thank the financial support of the project Centro-01-0145-FEDER-000017 EMaDeS - Energy, Materials and Sustainable Development, co-financed by the Portugal 2020 Program (PT 2020), within the Regional Operational Program of the Center (CENTRO 2020) and the European Union through the European Regional 
Development Fund (ERDF). JO wish to thank the FCT for the SFRH/BD/98219/2013 grant. The authors also acknowledge funding by the Spanish Ministry of Economy and Competitiveness (MINECO) through the project MAT2016-76039-C4-3-R

(AEI/FEDER, UE) (including the FEDER financial support), and financial support from the Basque Government Industry Department under the ELKARTEK Program.

\section{References}

[1] Soldano C. Hybrid metal-based carbon nanotubes: Novel platform for multifunctional applications. Progress in Materials Science. 2015;69:183-212.

[2] Mittal G, Dhand V, Rhee KY, Park S-J, Lee WR. A review on carbon nanotubes and graphene as fillers in reinforced polymer nanocomposites. Journal of Industrial and Engineering Chemistry. 2015;21:11-25.

[3] Georgakilas V, Gournis D, Tzitzios V, Pasquato L, Guldi DM, Prato M. Decorating carbon nanotubes with metal or semiconductor nanoparticles. Journal of Materials Chemistry. 2007;17(26):2679-94.

[4] Kharisov BI, Kharissova OV, Ortiz Méndez U, De La Fuente IG. Decoration of Carbon Nanotubes With Metal Nanoparticles: Recent Trends. Synthesis and Reactivity in Inorganic, Metal-Organic, and Nano-Metal Chemistry. 2016;46(1):55-76.

[5] Li L-H, Zhang W-D. Preparation of carbon nanotubes supported platinum nanoparticles by an organic colloidal process for nonenzymatic glucose sensing. Microchimica Acta. 2008;163(3-4):305-11.

[6] Dobrzański L, Pawlyta M, Krztoń A, Liszka B, Labisz K. Synthesis and characterization of carbon nanotubes decorated with platinum nanoparticles. Journal of Achievements in Materials and Manufacturing Engineering. 2010;39(2):184-9.

[7] Sharma P, Sharma RK. Asymmetric hydrogenation of $\alpha$-ketoesters on the $\operatorname{Pt}(111)$ surface. New Journal of Chemistry. 2016;40(11):9038-41.

[8] Sharma P, Sharma RK. Platinum functionalized multiwall carbon nanotube composites as recyclable catalyst for highly efficient asymmetric hydrogenation of methyl pyruvate. RSC Advances. 2015;5(124):102481-7.

[9] Ravindra R, Bhat BR. Hydrogen storage in palladium decorated carbon nanotubes prepared by solventless method. International Journal of Applied Physics and Mathematics. 2012;2(1):63.

[10] Bittencourt C, Felten A, Ghijsen J, Pireaux JJ, Drube W, Erni R, et al. Decorating carbon nanotubes with nickel nanoparticles. Chemical Physics Letters. 2007;436(46):368-72.

[11] Wen F, Zhang F, Liu Z. Investigation on microwave absorption properties for multiwalled carbon nanotubes/Fe/Co/Ni nanopowders as lightweight absorbers. The Journal of Physical Chemistry C. 2011;115(29):14025-30.

[12] Wu N, Lv H, Liu J, Liu Y, Wang S, Liu W. Improved electromagnetic wave absorption of Co nanoparticles decorated carbon nanotubes derived from synergistic magnetic and dielectric losses. Physical Chemistry Chemical Physics.

2016;18(46):31542-50.

[13] Hsieh C-T, Lin J-Y, Wei J-L. Deposition and electrochemical activity of Pt-based bimetallic nanocatalysts on carbon nanotube electrodes. International Journal of Hydrogen Energy. 2009;34(2):685-93. 
[14] Acierno S, Barretta R, Luciano R, Marotti de Sciarra F, Russo P. Experimental evaluations and modeling of the tensile behavior of polypropylene/single-walled carbon nanotubes fibers. Composite Structures. 2017;174:12-8.

[15] Patti A, Barretta R, Marotti de Sciarra F, Mensitieri G, Menna C, Russo P. Flexural properties of multi-wall carbon nanotube/polypropylene composites: Experimental investigation and nonlocal modeling. Composite Structures. 2015;131:282-9.

[16] Costa P, Silva J, Ansón-Casaos A, Martinez MT, Abad MJ, Viana J, et al. Effect of carbon nanotube type and functionalization on the electrical, thermal, mechanical and electromechanical properties of carbon nanotube/styrene-butadiene-styrene composites for large strain sensor applications. Composites Part B: Engineering.

2014;61(Supplement C):136-46.

[17] Cha J, Jun GH, Park JK, Kim JC, Ryu HJ, Hong SH. Improvement of modulus, strength and fracture toughness of CNT/Epoxy nanocomposites through the functionalization of carbon nanotubes. Composites Part B: Engineering. 2017;129(Supplement C):169-79.

[18] Carabineiro SAC, Pereira MFR, Pereira JN, Caparros C, Sencadas V, LancerosMendez S. Effect of the carbon nanotube surface characteristics on the conductivity and dielectric constant of carbon nanotube/poly(vinylidene fluoride) composites. Nanoscale Research Letters. 2011;6(1):X1-5.

[19] Costa P, Silva J, Lanceros Mendez S. Strong increase of the dielectric response of carbon nanotube/poly(vinylidene fluoride) composites induced by carbon nanotube type and pre-treatment. Composites Part B: Engineering. 2016;93:310-6.

[20] Ribeiro C, Sencadas V, Correia DM, Lanceros-Méndez S. Piezoelectric polymers as biomaterials for tissue engineering applications. Colloids and Surfaces B:

Biointerfaces. 2015;136:46-55.

[21] Costa CM, Silva MM, Lanceros-Méndez S. Battery separators based on vinylidene fluoride (VDF) polymers and copolymers for lithium ion battery applications. RSC Advances. 2013;3(29):11404-17.

[22] Nunes-Pereira J, Sencadas V, Correia V, Cardoso VF, Han W, Rocha JG, et al. Energy harvesting performance of $\mathrm{BaTiO}_{3} /$ poly(vinylidene fluoride-trifluoroethylene) spin coated nanocomposites. Composites Part B: Engineering. 2015;72:130-6.

[23] Xin Y, Sun H, Tian H, Guo C, Li X, Wang S, et al. The use of polyvinylidene fluoride (PVDF) films as sensors for vibration measurement: A brief review.

Ferroelectrics. 2016;502(1):28-42.

[24] Martins P, Lopes AC, Lanceros-Mendez S. Electroactive phases of poly(vinylidene fluoride): Determination, processing and applications. Progress in Polymer Science. 2014;39(4):683-706.

[25] Ferreira JCC, Monteiro TS, Lopes AC, Costa CM, Silva MM, Machado AV, et al. Variation of the physicochemical and morphological characteristics of solvent casted poly(vinylidene fluoride) along its binary phase diagram with dimethylformamide. Journal of Non-Crystalline Solids. 2015;412:16-23.

[26] De Almeida JMMM, Agostinho Moreira J. Influence of diffusion parameters on the spectral characteristics of raman modes of titanium-diffused lithium niobate planar waveguides. Spectroscopy Letters. 2013;46(6):453-8.

[27] Benz M, Euler WB. Determination of the crystalline phases of poly(vinylidene fluoride) under different preparation conditions using differential scanning calorimetry and infrared spectroscopy. Journal of Applied Polymer Science. 2003;89(4):1093-100. [28] Bedolla-Valdez ZI, Verde-Gómez Y, Valenzuela-Muñiz AM, Gochi-Ponce Y, Oropeza-Guzmán MT, Berhault G, et al. Sonochemical synthesis and characterization 
of $\mathrm{Pt} / \mathrm{CNT}, \mathrm{Pt} / \mathrm{TiO}_{2}$, and $\mathrm{Pt} / \mathrm{CNT} / \mathrm{TiO}_{2}$ electrocatalysts for methanol electro-oxidation. Electrochimica Acta. 2015; 186:76-84.

[29] Lopes AC, Caparros C, Ferdov S, Lanceros-Mendez S. Influence of zeolite structure and chemistry on the electrical response and crystallization phase of poly(vinylidene fluoride). Journal of Materials Science. 2013;48(5):2199-206.

[30] Dresselhaus MS, Jorio A, Souza Filho AG, Saito R. Defect characterization in graphene and carbon nanotubes using Raman spectroscopy. Philosophical Transactions of the Royal Society A: Mathematical, Physical and Engineering Sciences.

2010;368(1932):5355-77.

[31] Panchakarla LS, Govindaraj A, Rao CNR. Nitrogen- And boron-doped doubleWalled carbon nanotubes. ACS Nano. 2007;1(5):494-500.

[32] Bokobza L, Zhang J. Raman spectroscopic characterization of multiwall carbon nanotubes and of composites. Express Polymer Letters. 2012;6(7):601-8.

[33] Higgins DC, Meza D, Chen Z. Nitrogen-doped carbon nanotubes as platinum catalyst supports for oxygen reduction reaction in proton exchange membrane fuel cells. Journal of Physical Chemistry C. 2010;114(50):21982-8.

[34] He XN, Gao Y, Mahjouri-Samani M, Black PN, Allen J, Mitchell M, et al. Surface-enhanced Raman spectroscopy using gold-coated horizontally aligned carbon nanotubes. Nanotechnology. 2012;23(20):205702.

[35] Caparros C, Lopes AC, Ferdov S, Lanceros-Mendez S. $\gamma$-Phase nucleation and electrical response of poly(vinylidene fluoride)/microporous titanosilicates composites. Materials Chemistry and Physics. 2013;138(2-3):553-8.

[36] Nunes-Pereira J, Costa CM, Leones R, Silva MM, Lanceros-Méndez S. Li-ion battery separator membranes based on poly(vinylidene fluoride-

trifluoroethylene)/carbon nanotube composites. Solid State Ionics. 2013;249-250:63-71. [37] Costa P, Silva J, Sencadas V, Costa CM, van Hattum FWJ, Rocha JG, et al. The effect of fibre concentration on the $\alpha$ to $\beta$-phase transformation, degree of crystallinity and electrical properties of vapour grown carbon nanofibre/poly(vinylidene fluoride) composites. Carbon. 2009;47(11):2590-9.

[38] Avouris P, Freitag M, Perebeinos V. Carbon-nanotube photonics and optoelectronics. Nat Photon. 2008;2(6):341-50.

[39] Carabineiro SAC, Pereira MFR, Nunes-Pereira J, Silva J, Caparros C, Sencadas V, et al. The effect of nanotube surface oxidation on the electrical properties of multiwall carbon nanotube/poly(vinylidene fluoride) composites. Journal of Materials Science. 2012;47(23):8103-11.

[40] Ferreira A, Martínez MT, Ansón-Casaos A, Gómez-Pineda LE, Vaz F, LancerosMendez S. Relationship between electromechanical response and percolation threshold in carbon nanotube/poly(vinylidene fluoride) composites. Carbon. 2013;61:568-76.

[41] Charlier J-C, Blase X, Roche S. Electronic and transport properties of nanotubes. Reviews of Modern Physics. 2007;79(2):677-732. 